Research article

\title{
Dose-dependent effects of isoflurane on TrkB and GSK3 $\beta$ signaling: Importance of burst suppression pattern
}

\author{
Wiebke Theilmann ${ }^{1,3}$, Okko Alitalo ${ }^{3}$, Iris Yorke ${ }^{2}$, Tomi Rantamäki* \\ Laboratory of Neurotherapeutics, Molecular and Integrative Biosciences Research Programme, Faculty of Biological and Environmental Sciences, and Division of \\ Pharmacology and Pharmacotherapeutics, Faculty of Pharmacy, ! niversity of " elsin\#i, Finland
}

\section{GRAPHICALA B STRACT}

Brief burst-suppressing isoflurane anesthesia have been shown to produce antidepressant effects in patients, and in mice to bring phosphorylation effects on TrkB receptor and GSK3 $\beta$ kinase similar to those seen with rapid-acting antidepressant ketamine. We investigated whether the emergence of burst-suppression pattern, a neurophysiological correlate of deep anesthesia, is required for these molecular changes induced by isoflurane. Isoflurane dose-dependently regulates TrkB and GSK3 $\beta$ phosphorylation and most prominent changes are observed when EEG burst suppression emerge, thus with a dosing associated with antidepressant effects.

\section{A R T I CLE I N F O}

\section{\$ ey\%ords\&}

Anesthesia

Protein phosphorylation

EEG burst suppression

Electrocerebral silence

Antidepressant

\begin{abstract}
A B STR A C T
' b(ectives\& Deep burst-suppressing isoflurane anesthesia regulates signaling pathways connected with antidepressant responses in the rodent brain: activation of TrkB neurotrophin receptor and inhibition of GSK3 $\beta$ kinase (glycogen synthase kinase $3 \beta$ ). The main objective of this study was to investigate whether EEG (electroencephalogram) burst suppression correlates with these intriguing molecular alterations induced by isoflurane.

Methods\& Adult male mice pre-implanted with EEG recording electrodes were subjected to varying concentrations of isoflurane (1.0-2.0\% ad $20 \mathrm{~min})$ after which medial prefrontal cortex samples were collected for molecular analyses, and the data retrospectively correlated to EEG (+ / - burst suppression).

Results\& Isoflurane dose-dependently increased phosphorylation of TrkB ${ }^{\mathrm{Y} 816}, \mathrm{CREB}^{\mathrm{S133}}$ (CAMP response element binding protein), GSK3 $\beta^{S 9}$ and $p 70 S 6 k^{T 412 / S 424}$. The time spent in burst suppression mode varied considerably between individual animals. Notably, a subset of animals subjected to $1.0-1.5 \%$ isoflurane showed no burst
\end{abstract}

\footnotetext{
* Corresponding author.

E)mail address\&tomi.rantamaki@helsinki.fi (T. Rantamäki).

${ }^{1}$ Present address: Institute of Pharmacology, Toxicology and Pharmacy, University of Veterinary Medicine Hannover, Germany.

2 Present address: Orion Corporation ORION PHARMA, Clinical Data Management, Clinical Operations, Research \& Development, Orionintie 1, P O Box 65, FI02101 Espoo, Finland.

${ }^{3}$ These author equally contributed to this work.
} 


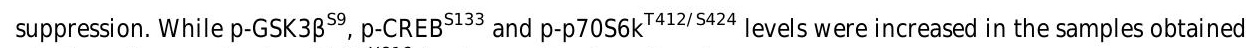
also from these animals, $\mathrm{p}$-TrkB ${ }^{\mathrm{Y} 816}$ levels remained unaltered.

* onclusions\& Isoflurane dose-dependently regulates TrkB and GSK3 3 signaling and dosing associated with therapeutic outcomes in depressed patients produces most prominent effects.

General anesthetics (GA), such as halogenated hydrocarbon isoflurane, are widely used in medical and experimental operations requiring surgical anesthesia. GAs strongly and rapidly alter brain's electrical activity and as the concentration of an anesthetic is increased so-called burst suppression state is eventually reached [1,2]. Alternating periods of low voltage EEG (electroencephalogram) activity and high-voltage electrical bursting activity are characteristic for this state. The durations of suppressed periods are prolonged as anesthesia further deepens, finally progressing into intermittent periods of isoelectric EEG (i.e. electrocerebral silence). Notably, these neurophysiological signs of deep anesthesia can be achieved with remarkably similar dosing of volatile and gaseous anesthetics in mice and men.

The efficacy and onset of action of electroconvulsive therapy (ECT), among the most potent antidepressant in the clinical domain, have been associated with post-ictal (i.e. after seizure) emergence of burst suppression EEG pattern $[3,4]$. This encouraged early investigations to test the intriguing possibility that mere "electrocerebral silence" induced by GAs produces antidepressant effects equal to those produced by the ECT. While some of these clinical studies, especially those carried out with isoflurane, appeared promising also conflicting results were reported [5-7]. We recently translated this treatment into the preclinical context and demonstrated that isoflurane anesthesia rapidly regulates some of the key molecular signaling events implicated in antidepressant responses. Similarly to that observed with pharmacologically diverse classical antidepressant drugs [8-10], autophosphorylation of TrkB (the receptor for brain-derived neurotrophic factor or BDNF) and phosphorylation of $\mathrm{CREB}^{\mathrm{S133}}$ (CAMP response element binding protein) were readily and rapidly increased in the adult rodent medial prefrontal cortex upon exposure to anesthetic concentrations of isoflurane [11]. Isoflurane also increased the phosphorylation of $p 70 \mathrm{~S} 6 \mathrm{k}^{\mathrm{T} 412 / \mathrm{S} 424}$ and GSK $3 \beta^{\text {S9 }}$ (glycogen synthase kinase $3 \beta$ ) [11]. These molecular effects resemble those induced by rapid-acting antidepressant ketamine $[12,13]$. While we also demonstrated that a subanesthetic dose of isoflurane $(0.3 \%)$ showed no such effects [11], the correlation between the emergence of burst suppression pattern and TrkB and GSK3 $\beta$ phosphorylation alterations induced by isoflurane have not yet been investigated within the same animals. The main purpose of this study was to test this hypothesis.

\section{,,+- . nimals}

Altogether 18 male C57BL/ 6J RccHsd mice (8-week-old, 24-26 g, Harlan Laboratories, Venray, Netherlands) were used in the study. Animals were maintained under standard laboratory conditions (12hour light-dark cycle, temperature $20 \pm 1{ }^{\circ} \mathrm{C}$, humidity 60-70\%) with free access to food and water. Mice were housed in groups of four until surgery and individually thereafter. Nesting and gnawing material was provided and the cages were enriched with "mouse houses". Group sizes were determined on the basis of expected effect size and variation observed in our three recent publications wherein essentially similar isoflurane dosing protocol was used [11,14,15]. Food and water availability, room temperature, humidity and animal wellbeing were checked daily. Bedding material was changed once per week. After surgery, bodyweight was measured regularly to monitor weight gain. Exclusion criteria were poor body condition (no grooming, hunched posture, reduced/no motor activity) and weight loss $>20 \%$. None of the animals were excluded from the experiment. All efforts were made to minimize pain or discomfort of the animals. Experiments were done in compliance with the European Communities Council Directive of 24 November 1986 (86/609/EEC) and were approved by the County Administrative Board of Southern Finland (License nro: ESAVI/ 10527/ 04.10.07/2014).

\section{,,++ Implantation of $\mathrm{EE} / \mathrm{OEM} /$ electrodes}

For the implantation of EEG/EMG-electrodes, all mice were anesthetized with isoflurane $(3.0 \%$ induction, $1.5-2.0 \%$ maintenance (Vetflurane, Virbac, Switzerland)) and locally with lidocaine (10 mg/ $\mathrm{ml}$; Orion Pharma, Finland). Buprenorphine- $\mathrm{HCl}(0.1 \mathrm{mg} / \mathrm{kg}$, s.c.,; Indivior, USA) was used for postoperative care. Two epidural screw EEG electrodes were placed above the fronto-parietal cortex. A further screw served as mounting support. Two silver wire electrodes were implanted in the nuchal muscles to monitor the EMG. After surgery, mice were single-housed in Plexiglas boxes. After a recovery period of one week, animals were connected to flexible counterbalanced cables for EEG/EMG recording and habituated to recording cables for 3 days.

\section{,+ 1 , Pharmaco)EE/ study and dissection of brain samples}

Animals were assigned randomly to treatment groups by drawing lots: $1 \%$ isoflurane anesthesia $(n=5), 1.5 \%$ isoflurane anesthesia $(n=5), 2 \%$ isoflurane anesthesia $(n=4)$ and sham $(n=4)$. Mice were exposed to isoflurane (Vetflurane, Virbac, Switzerland) in their home cage for total period of $20 \mathrm{~min}$. Airflow was $0.5-0.8 \mathrm{l} / \mathrm{min}$. Sham animals were kept in their home cages without isoflurane. Animals were euthanized immediately after the treatments using rapid cervical dislocation and samples from medial prefrontal cortex collected as previously described $[11,16]$ by a researcher blinded to experimental groups. All experiments were performed between 9:00 - 12:00 A.M. Samples were stored at $-80^{\circ} \mathrm{C}$ until analysis.

\section{,+ 2 , EE/ analysis}

The EEG and EMG signals were amplified (gain 5 or $10 \mathrm{~K}$ ) and filtered (high pass: $1 \mathrm{~Hz}$; low pass $100 \mathrm{~Hz}$ ) with a 16-channel AC amplifier (A-M System, model 3500), sampled at $254 \mathrm{~Hz}$ or $70 \mathrm{~Hz}$ with 1401 unit (CED), and recorded using Spike2 (version 8.07, Cambridge Electronic Devices). The EEG data were recorded and processed using Spike2 (version 6, Cambridge Electronic Devices). Bursting activity was detected semi-automatically with the aid of the "Get EEG bursts script" (kindly provided by Dr. Paul Shepard, University of Maryland School of Medicine) in Spike2. Burst suppression state was defined as a pattern of suppressed or absent EEG activity (suppression EEG amplitude $\leq 30 \%$ of baseline) alternated with periods of high amplitude events (burst EEG amplitude $\geq 130 \%$ of baseline).

\section{$+, 3,4$ estern blot}

Western blotting was performed as previously described [11,14,16]. Brain samples were homogenized in a lysis buffer $(137 \mathrm{mM} \mathrm{NaCl}$, $20 \mathrm{mM}$ Tris, $1 \% \mathrm{NP}-40,10 \%$ glycerol, $48 \mathrm{mM} \mathrm{NaF}, \mathrm{H}_{2} \mathrm{O}$, Complete inhibitor mix (Roche, Switzerland), PhosphoStop (Roche, Switzerland)), incubated on ice, centrifuged $\left(16000 \mathrm{~g}, 15 \mathrm{~min},+4{ }^{\circ} \mathrm{C}\right.$ ) and the resulting supernatant collected for further analysis. Sample protein 
concentrations were measured using Bio-Rad DC protein assay (Bio-Rad Laboratories, Hercules, USA). Samples ( $40 \mu$ g protein) were separated with SDS-PAGE under reducing conditions and blotted to a PVDF membrane. Membranes were incubated with the following primary antibodies (https:// scicrunch.org Research Identification Portal Identity Number (RRID) in brackets): anti-p-GSK3 $\beta^{\text {S9 }}$ (\#9336, RRID:AB 331405; 1:000, Cell Signaling Technology (CST)), anti-pTrkA $^{\text {Y785/TrkB }}{ }^{\text {Y816 }}$ (\#4168, RRID:AB_10620952; 1:000, CST), anti-pCREB $^{\text {S133 }}$ (\#9198, RRID:AB_2561044; 1:000, CST), anti-p-p70S6k ${ }^{T 412 /}$ S424 (\#9234, RRID:AB 2269803, 1:000, CST) and anti-GAPDH (\#2118, RRID:AB 561053; 1:000, CST). Further, the membranes were washed with TBS/ $0.1 \%$ Tween (TBST) and incubated with horseradish peroxidase conjugated secondary antibodies (1:10,000 in non-fat dry milk, $1 \mathrm{~h}$ at room temperature; Bio-Rad). After subsequent washes, secondary antibodies were visualized using enhanced chemiluminescence ( $\mathrm{ECL}$ Plus, ThermoScientific, Vantaa, Finland) for detection Bio-Rad ChemiDoc MP camera (Bio-Rad Laboratories, Helsinki, Finland). The data were normalized to GAPDH due to our previous observations that similar isoflurane treatment protocol does not affect total protein levels of investigated molecular targets [11]. The GAPDH levels were also not affected by isoflurane treatment (data not shown). Processing of the brain samples and analysis of the western blots were blinded to the identity of the treatment versus sham groups.

\section{,+ 6 , Statistical analyses}

Results are expressed as a percentage of the sham anesthesia and shown as mean \pm SEM (standard error of mean) and normalized to GAPDH loading control. Two-tailed Mann-Whitney test or one-way analysis of variance (ANOVA) (two categorical independent variables) followed by Tukey's multiple comparison test were used for statistical evaluation (Prism 7 software, GraphPad (La Jolla, CA, USA). A $P<0.05$ was considered statistically significant. No outlier test was used to discount any data point and no data were excluded from the analyses.

While the isoflurane dosing regimen utilized in our previous animal studies is expected to produce burst suppression pattern [14,15], the correlation between the emergence of burst suppression pattern and TrkB and GSK3 $\beta$ phosphorylation alterations induced by isoflurane have not yet been investigated within the same animals. To this end, we subjected adult male mice pre-implanted with EEG recording electrodes to varying concentrations of isoflurane for a period of $20 \mathrm{~min}$ after which samples from the medial prefrontal cortex were collected for molecular analyses (Fig. 1A). Isoflurane produced dose-dependent ef-

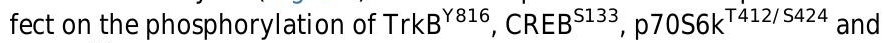
GSK $3 \beta^{S 9}$ and most prominent effects were seen with $2 \%$ isoflurane, also used in our previous studies (Fig. 1B [11-14]. Notably, and as also seen previously [11], the magnitude of these phosphorylation changes varied considerably. Phosphorylation of $\mathrm{CREB}^{\mathrm{S} 133}$ and $\mathrm{GSK} 3 \beta^{\mathrm{S9}}$ showed by and large the most prominent changes in response to isoflurane while phosphorylation of $\operatorname{TrkB}^{\mathrm{Y} 816}$ was less robustly increased compared to sham condition.

We retrospectively divided the animals into two groups based on their EEG signature while under the influence of isoflurane (burst suppression / no burst suppression) (Fig. 1C). In all of the animals exposed to highest isoflurane concentration (2.0\%), a burst suppression pattern was readily evident in the EEG, while burst suppression was

Isoflurane-induced TrkB and GSK3 $\beta$ phosphorylation - potential importance of EEG burst suppression. (A) Schematic representation of the study design. Adult male mice pre-implanted with recording electrodes were exposed to varying concentrations of isoflurane (1.0\%, $1.5 \%$ or $2.0 \%)$ for a period of 20 -minutes under EEG monitoring after which samples from the medial prefrontal cortex were collected for molecular analyses. Obtained data were correlated to EEG (burst-suppression or no burst-suppression) within the same animal. ( ) Dose-dependent effect of isoflurane on TrkB ${ }^{Y 816}\left(F_{1,-3}=551\right), C^{2} E^{S 133}\left(F_{1,-3}=1202\right), p 70 S 6 k^{\top 421 / 424}$ $\left(F_{1,-3}=2423\right)$, and GSK3 $\beta^{S 9}\left(F_{1,-3}=281\right)$ phosphorylation (sham $N=4,1 \%$ isoflurane $N=5,1.5 \%$ isoflurane $N=5,2 \%$ isoflurane $\left.N=4\right)$. $)$ Representative EEG

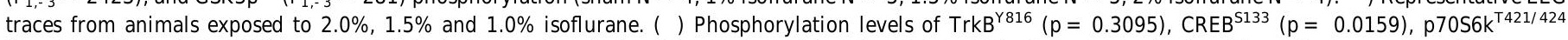
$(p=0.0079)$, and GSK3 $\beta^{59}(p=0.0952)$ in brain samples obtained from mice subjected to $1.0-1.5 \%$ isoflurane anesthesia without the emergence of burst suppression pattern $(\mathrm{N}=6)$ and sham $(\mathrm{N}=4)$. Full statistics are presented in $\quad . * p<0.05 ; * * p<0.01$; *** $p<0.005$, two-tailed MannWhitney test $(D)$ or one-way ANOVA followed by Tukey's multiple comparison test $(E)$. Abbreviations: $B S=$ burst suppression; $C R E B=C A M P$ response element binding protein; $E E G$ = electroencephalogram; GSK3 $\beta=$ glycogen synthase kinase $3 \beta ;$ Iso = isoflurane; $M W=$ molecular weight; Sham = mice in the induction chamber for 2 min with isoflurane flow; TrkB = tropomyosin related protein kinase $B$. 
essentially absent in a subgroup of mice subjected to $1.0 \%$ or $1.5 \%$ isoflurane ( $1.0 \%$ isoflurane: 4 out of $5 ; 1.5 \%$ isoflurane: 1 out of 5$)$ ( Fig. 1A).

To investigate whether the emergence of burst suppression pattern is prerequisite for the ability of isoflurane to regulate phosphorylation

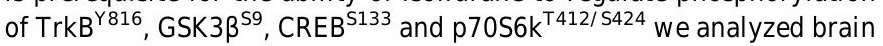
samples collected from the mice not displaying burst suppression under isoflurane administration and compared the results to the sham group. Importantly, phosphorylation of $\mathrm{p} 70 \mathrm{~S} \mathrm{k}^{\mathrm{T} 412 / \mathrm{S} 424}$ and $\mathrm{CREB}^{\mathrm{S} 133}$ were increased and GSK3 $\beta^{59}$ displayed a trend $(p=0.0952)$, while phosphorylation of $\mathrm{TrkB}^{\mathrm{Y} 816}$ remained unchanged (Fig. 1D). In sum, this report indicates that isoflurane dose-dependently regulates TrkB and GSK3 $\beta$ signaling and most prominent changes are seen with a dosing leading into EEG burst suppression (i.e. deep anesthesia). Burst suppression pattern varied significantly between individual animals, but the time spent in burst suppression state correlated with the increased

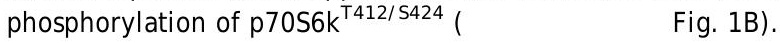

"

Activation of BDNF receptor TrkB and inhibition of GSK3 $\beta$ kinase have been associated with the mechanism of action of rapid-acting antidepressant ketamine $[17,18]$. Upon activation TrkB receptors undergo tyrosine phosphorylation within their intracellular domains [19] and phosphorylation of the autophosphorylation loop (Y705/6) and the phospholipase- $\mathrm{C} Y 1$ binding site $(\mathrm{Y} 816)$ are especially regulated by antidepressants [9]. In contrast, phosphorylation of the serine-9 residue reduces the kinase activity of GSK3 $\beta$ [20]. Our recent study demonstrates that brief but deep isoflurane anesthesia bring along these molecular effects [11], providing novel insights into the rapid antidepressant effects of isoflurane observed in a subset of depressed patients $[5,6,21]$. The rationale to investigate isoflurane in clinical trials lied upon the idea that post-ictal (i.e. after seizure) emergence of burst-suppression and/or electrocerebral silence predicts the onset-ofaction of ECT [3-6]. Unlike ECT, isoflurane reliably and reproducibly produces EEG burst suppression if adequate dosing regimen is used. Notably, acute electroconvulsive shock (ECS; an animal model of ECT) has not been shown to activate TrkB [22] although it strongly and rapidly increases BDNF synthesis [23]. Increased TrkB phosphorylation has been, however, reported after 10-day ECS treatment [24]. Yet, a single ECS does increase GSK $3 \beta^{59}$ phosphorylation gradually after seizure termination [25-27]. This report suggests that isoflurane regulates p70S6k and GSK3 $\beta$ phosphorylation at a relatively low dose. It is tempting to speculate that isoflurane might possess antidepressant properties already at subanesthetic/sedative doses, similarly to that seen with ketamine [28] and more recently with nitrous oxide $\left(\mathrm{N}_{2} \mathrm{O}\right.$, laughing gas) [[29]29, see also ref. [16]16]. On the other hand, the most prominent effects were observed with a dosing leading into EEG burst suppression, indicating that the depth of anesthesia (and ongoing metabolic alterations) are somehow coupled with TrkB and GSK3 $\beta$ signaling. Notably, we recently demonstrated that $\mathrm{N}_{2} \mathrm{O}$ and flurothyl (a treatment reminiscent to ECT) regulates TrkB and GSK3 $\beta$ phosphorylation only upon drug withdrawal during a brain state dominated by slow EEG oscillations that are characteristic for deep sleep [16].

Several drugs, including mood stabilizer lithium (chemical element; e.g. an inhibitor of inositol monophosphate and GSK3ß) [30], classical antidepressant drugs (e.g. monoamine reuptake blockers) [8,9,31], nimodipine (an L-type calcium channel blocker) [32], 7,8-dihydroxyflavone (an antioxidant) [33], donepezil (an acetylcholine esterase inhibitor) [34], and anesthetics such as ketamine (an N-methyl-Daspartate (NMDA) receptor blocker) $[10,14]$ and isoflurane (a GABA receptor agonist) [11,14] have been shown to rapidly phosphorylate TrkB in the adult rodent brain. Among these agents, at least ketamine, tricyclic antidepressants, lithium and GAs also rapidly increase GSK3 ${ }^{\text {S9 }}$ phosphorylation $[11,14,15,27,35]$. The pharmacological actions and effects of these pharmacological compounds are very distinct implying that several receptor-level mechanisms can drive these seemingly similar effects on TrkB and GSK3 $\beta$ signaling. Indeed, while ketamine is thought to regulate TrkB signaling through BDNF dependent mechanism [10] the precise mechanism underlying the effects of classical antidepressants and isoflurane remain poorly understood but seems not to involve BDNF $[11,31,36]$. Whether these molecular responses driven through putatively different mechanisms share other characteristics and functional after effects remain poorly studied. It is currently unclear whether classical antidepressants and rapid-acting antidepressants regulate TrkB and GSK3 $\beta$ signaling even in the same cell populations. High-precision localization studies require, however, the use of fixed samples for immunohistochemical analysis that must be prepared using anesthesia during the fixation step. Indeed, this report adds further evidence on the earlier literature demonstrating that anesthesia, that is routinely employed in experimental research involving laboratory rodents, produce rather profound changes on intracellular signaling in the brain [14].

A major advance of our experiment set-up was that molecular level alterations and EEG were analyzed within the same animals. We are utilizing similar strategy complemented with in-depth time-lapsed EEG analysis aiming at identifying potential shared EEG signatures correlating with ongoing TrkB and GSK3 $\beta$ signaling alterations induced by mechanistically distinct treatments carrying rapid antidepressant potential (e.g. subanesthetic ketamine, ECS, isoflurane, $\mathrm{N}_{2} \mathrm{O}$ ) (see Ref. [16]).

In sum, this brief report demonstrates that isoflurane dose-dependently regulates TrkB and GSK3 $\beta$ phosphorylation in the adult rodent brain and most prominent changes are observed when burst-suppression pattern in the EEG emerge.

\#

The authors report no conflict of interest.

\section{$\$ \% \&$}

Samuel Kohtala is thanked for technical assistance. Dr. Paul Shepard is thanked for providing the script for Spike2. Dr. Tarja Stenberg and Dr. Henna-Kaisa Wigren are thanked for their support and advise. This study has been supported by the Academy of Finland under Grants 276333, 284569, 305195 and 312664.

\section{$\$ \quad, \$$}

Supplementary material related to this article can be found, in the online version, at doi:https:// doi.org/ 10.1016/j.neulet.2018.11.018.

\#

[1] F. Amzica, What does burst suppression really mean? Epilepsy Behav. 49 (2015) 234-237.

[2] E.N. Brown, R. Lydic, N.D. Schiff, General Anesthesia, Sleep, and Coma, N. Engl. J. Med. 363 (2010) 2638-2650.

[3] A.D. Krystal, R.D. Weiner, C.E. Coffey, The ictal EEG as a marker of adequate stimulus intensity with unilateral ECT, J. Neuropsychiatry Clin. Neurosci. 7 (1995) 295-303.

[4] M.S. Nobler, et al., EEG manifestations during ECT: effects of electrode placement and stimulus intensity, Biol. Psychiatry 34 (1993) 321-330.

[5] G. Langer, J. Neumark, G. Koinig, M. Graf, G. Schönbeck, Rapid psychotherapeutic effects of anesthesia with isoflurane (ES narcotherapy) in treatment-refractory depressed patients, Neuropsychobiology 14 (1985) 118-120.

[6] G. Langer, et al., Isoflurane narcotherapy in depressive patients refractory to conventional antidepressant drug treatment. A double-blind comparison with electroconvulsive treatment, Neuropsychobiology 31 (1995) 182-194.

[7] W. Engelhardt, G. Carl, E. Hartung, Intra-individual open comparison of burstsuppression-isoflurane-anaesthesia versus electroconvulsive therapy in the treatment of severe depression, Eur. I. Anaesthesiol. 10 (1993) 113-118.

[8] T. Saarelainen, et al., Activation of the TrkB Neurotrophin Receptor Is Induced by Antidepressant Drugs and Is Required for Antidepressant-Induced Behavioral Effects, J. Neurosci. 23 (2003) 349-357.

[9] T. Rantamäki, et al., Pharmacologically diverse antidepressants rapidly activate 
brain-derived neurotrophic factor receptor TrkB and induce phospholipaseCgamma signaling pathways in mouse brain, Neuropsychopharmacol. Off. Publ. Am. Coll. Neuropsychopharmacol. 32 (2007) 2152-2162.

[10] A.E. Autry, et al., NMDA receptor blockade at rest triggers rapid behavioural antidepressant responses, Nature 475 (2011) 91-95.

[11] H. Antila, et al., Isoflurane produces antidepressant effects and induces TrkB signaling in rodents, Sci. Rep. 7 (2017) 7811.

[12] N. Li, et al., mTOR-dependent synapse formation underlies the rapid antidepressant effects of NMDA antagonists, Science 329 (2010) 959-964.

[13] E. Beurel, L. Song, R. J ope, Inhibition of glycogen synthase kinase-3 is necessary for the rapid antidepressant effect of ketamine in mice, Mol. Psychiatry 16 (2011) 1068-1070.

[14] S. Kohtala, et al., Brief isoflurane anesthesia produces prominent phosphoproteomic changes in the adult mouse hippocampus, ACS Chem. Neurosci. (2016), https:// doi. org/ 10.1021/acschemneuro.6b00002.

[15] J.V. Leikas, et al., Brief isoflurane anesthesia regulates striatal AKT-GSK3 $\beta$ signaling and ameliorates motor deficits in a rat model of early-stage Parkinson's disease, J . Neurochem. (2017), https:// doi.org/ 10.1111/jnc.14066.

[16] S. Kohtala, et al., Cortical Excitability and Activation of TrkB Signaling During Rebound Slow Oscillations Are Critical for Rapid Antidepressant Responses, Mol. Neurobiol. (2018), https:// doi.org/ 10.1007/ s12035-018-1364-6.

[17] R.S. Duman, G.K. Aghajanian, Synaptic dysfunction in depression: potential therapeutic targets, Science 338 (2012) 68-72.

[18] T. Rantamäki, I. Yalcin, Antidepressant drug action - from rapid changes on network function to network rewiring, Prog. Neuropsychopharmacol. Biol. Psychiatry 64 (2016) 285-292.

[19] R.A. Segal, et al., Differential Utilization of Trk Autophosphorylation Sites, J. Biol. Chem. 271 (1996) 20175-20181.

[20] R. Ilouz, S. Pietrokovski, M. Eisenstein, H. Eldar-Finkelman, New insights into the autoinhibition mechanism of glycogen synthase kinase-3beta, 」. Mol. Biol. 383 (2008) 999-1007.

[21] H.R. Weeks IIlet al., Antidepressant and neurocognitive effects of isoflurane anesthesia versus electroconvulsive therapy in refractory depression, PLoS One 8 (2013) e69809.

[22] H.H. Hansen, et al., Rapid Activation of the Extracellular Signal-Regulated Kinase $1 / 2$ (ERK1/2) Signaling Pathway by Electroconvulsive Shock in the Rat Prefronta Cortex Is Not Associated with TrkB Neurotrophin Receptor Activation, Cell. Mol. Neurobiol. 27 (2007) 585-594.

[23] M. Nibuya, S. Morinobu, R.S. Duman, Regulation of BDNF and trkB mRNA in rat brain by chronic electroconvulsive seizure and antidepressant drug treatments, J . Neurosci. Off. J. Soc. Neurosci. 15 (1995) 7539-7547.

[24] S. Enomoto, et al., Activated brain-derived neurotrophic factor/TrkB signaling in rat dorsal and ventral hippocampi following 10-day electroconvulsive seizure treatment, Neurosci. Lett. 660 (2017) 45-50.

[25] U.G. Kang, et al., Activation of protein kinase B (Akt) signaling after electroconvulsive shock in the rat hippocampus, Prog. Neuropsychopharmacol. Biol. Psychiatry 28 (2004) 41-44.

[26] K. Basar, E. Eren-Kocak, H. Ozdemir, A. Ertugrul, Effects of acute and chronic electroconvulsive shocks on glycogen synthase kinase $3 \beta$ level and phosphorylation in mice, J. ECT 29 (2013) 265-270.

[27] M.-S. Roh, et al., Biphasic changes in the Ser-9 phosphorylation of glycogen synthase kinase-3beta after electroconvulsive shock in the rat brain, Prog. Neuropsychopharmacol. Biol. Psychiatry 27 (2003) 1-5.

[28] R.M. Berman, et al., Antidepressant effects of ketamine in depressed patients, Biol. Psychiatry 47 (2000) 351-354.

[29] P. Nagele, et al., Nitrous oxide for treatment-resistant major depression: a proof-ofConcept trial, Biol. Psychiatry 78 (2015) 10-18.

[30] T. Rantamäki, J.E.A. Knuuttila, M.-E. Hokkanen, E. Castrén, The effects of acute and long-term lithium treatments on trkB neurotrophin receptor activation in the mouse hippocampus and anterior cingulate cortex, Neuropharmacology 50 (2006) 421-427.

[31] T. Rantamäki, et al., Antidepressant drugs transactivate TrkB neurotrophin receptors in the adult rodent brain independently of BDNF and monoamine transporter blockade, PLoS One 6 (2011) e20567.

[32] J. Koskimäki, N. Matsui, ] . Umemori, T. Rantamäki, E. Castrén, Nimodipine Activates TrkB Neurotrophin Receptors and Induces Neuroplastic and Neuroprotective Signaling Events in the Mouse Hippocampus and Prefrontal Cortex Cell. Mol. Neurobiol. (2014), https:// doi.org/ 10.1007/ s10571-014-0110-5.

[33] S.-W. J ang, et al., A selective TrkB agonist with potent neurotrophic activities by 7,8-dihydroxyflavone, Proc. Natl. Acad. Sci. U. S. A. 107 (2010) 2687-2692.

[34] H. Autio, et al., Acetylcholinesterase inhibitors rapidly activate Trk neurotrophin receptors in the mouse hippocampus, Neuropharmacology 61 (2011) 1291-1296.

[35] E. Beurel, M.A. Mines, L. Song, R.S. J ope, Glycogen synthase kinase-3 levels and phosphorylation undergo large fluctuations in mouse brain during development, Bipolar Disord. 14 (2012) 822-830.

[36] A. Di Lieto, et al., The Responsiveness of TrkB to BDNF and Antidepressant Drugs Is Differentially Regulated during Mouse Development, PLoS One 7 (2012). 